This is the peer-reviewed version of the paper:

Radonjic, A., 2020. Integer Codes Correcting Double Errors and Triple-Adjacent Errors Within a Byte. IEEE Transactions on Very Large Scale Integration (VLSI) Systems 28, 1901-1908.

https://doi.org/10.1109/TVLSI.2020.2998364

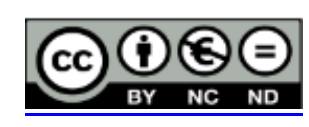

This work is licensed under the Attribution-NonCommercial-NoDerivatives 4.0 International (CC BY-NC-ND 4.0) 


\title{
Integer Codes Correcting Double Errors and Triple-Adjacent Errors within a Byte
}

\author{
Aleksandar Radonjic
}

\begin{abstract}
This paper presents a class of integer codes that are suitable for use in optical computer networks where the data is transmitted serially. The presented codes are constructed with the help of a computer and have three desirable properties. First, they use integer and lookup table operations, which makes them suitable for software implementation. Second, depending on the application requirements, the proposed codes can be used as lower rate error correction (EC) codes or as high-rate error detection (ED) codes. In the EC mode, which is suited for realtime applications, the receiver can correct all single and double errors as well as all triple-adjacent (TA) errors within one $b$-bit byte. On the other hand, if the integrity of data is of high importance, the receiver may operate in the ED mode. In that case, it is able to detect all quadruple errors, all double TA errors within one $b$-bit byte and all double TA errors within two $b$-bit bytes. Finally, it is important to note that the presented codes can be interleaved without delay and without using any additional hardware. Owing to this, it is possible to construct simple codes capable of detecting/correcting multiple $\mathrm{TA}$ and random errors.
\end{abstract}

Index Terms-Integer codes, optical computer networks, error control, single errors, double errors, triple-adjacent errors.

\section{INTRODUCTION}

Optical computer networks (OCNs) are usually classified in terms of the area they cover. Thus, for example, optical LANs (local area networks) connect computers that are physically close together, optical MANs (metropolitan area networks) are used to connect optical LANs, while optical WANs (wide area networks) are designed to connect computers on a national or continental scale (Fig. 1a) [1]. Due to these differences, these networks operate at different speeds: optical LANs and MANs typically run at $1 \mathrm{Gbps}$ or $10 \mathrm{Gbps}$, while optical WANs rarely have data rates above $1 \mathrm{Gbps}$ (these networks are mostly built using leased lines) [1], [2].

Although these facts are well documented in the literature, it is very rarely mentioned that OCNs share several common features. The first of them concerns the high computing power of the network nodes (NNs). Namely, from [3] it is known that exterior nodes (computers and servers) are always equipped with general purpose processors (GPPs), while interior nodes (routers and switches) contain network processors (NPs). The only difference between these chips is that NPs integrate some additional coprocessors [3] (e.g. for cryptographic operations). Hence, it can be said that each NN has hardware to perform integer, floating-point, logical and memory-based operations (e.g. lookup table (LUT) operations).

The author is with the Institute of Technical Sciences of the Serbian Academy of Sciences and Arts, 11000 Belgrade, Serbia.
The second common characteristic of almost all OCNs is that they use Ethernet packets for internode communication. In optical LANs/MANs these packets are used directly, while in optical WANs they are encapsulated into different frames (e.g. GFP frame [2]). Each Ethernet packet, as it is known, contains between 368 and 12000 bits of data, and ends with a 32-bit $\mathrm{CRC}$ code. Owing to this, the receiver is able to detect up to three random errors and all bursts of length up to 32 bits.

The third and last common feature relates to the behaviour of channel errors. In many studies, such as [4]-[8], it has been shown that single errors are 10 to 1000 times more frequent than any other type of errors. Besides this, in [4]-[8] it was shown that the burst error probability decreases exponentially with increasing burst length (Fig. 1b). If we add to this the fact that some OCNs may have high error rates (between $10^{-4}$ and $10^{-3}$ ) [7] we easily come to the conclusion that the most likely errors may occur twice. Therefore, it is reasonable to assume that, in OCNs, the vast majority of corrupted packets will contain either single, double or triple-adjacent (TA) errors.

Bearing this in mind, one may conclude that $\mathrm{CRC}$ codes are not the optimal solution for OCNs. First of all, these codes are based on finite field (FF) arithmetic, which is not supported by GPPs. For this reason, all NNs are equipped with hardware that performs FF operations. The second disadvantage is that CRCs cannot efficiently adapt to the application requirements. Namely, they are designed to detect the errors, but not to correct them. This means that each erroneous packet will be recovered through retransmission, which may be unacceptable for real-time (RT) applications.

Motivated by these facts, in this paper, we extend our previous work [9]-[14], and present a class of integer codes suitable for use in OCNs where the data is transmitted serially. Compared to CRCs, our codes have three advantages. First, they use integer and LUT operations, which makes them suitable for implementation in software. Second, depending on the application requirements, the presented codes can be used as high-rate error detection (ED) codes or as lower rate error correction (EC) codes. In the EC mode, which is suited for RT applications, the receiver can correct all single and double errors, as well as all TA errors confined to one $b$-bit byte $\left(\mathrm{TA}_{1}\right.$ errors). On the other hand, if the integrity of data is of high importance, the receiver may operate in the ED mode. In that case, it is able to detect all quadruple errors, all double TA errors corrupting one $b$-bit byte (DTA 1 errors) and all double TA errors corrupting two $b$-bit bytes (DTA 2 errors). Finally, the codes presented in this paper can be interleaved without delay and without using any additional hardware. Owing to this, it is possible to construct very simple codes capable of correcting/detecting multiple TA and random errors. 


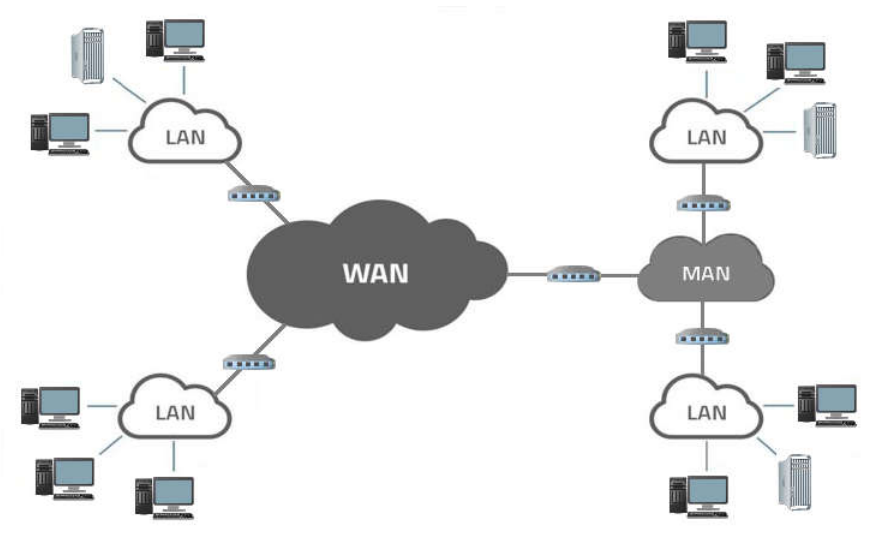

a)

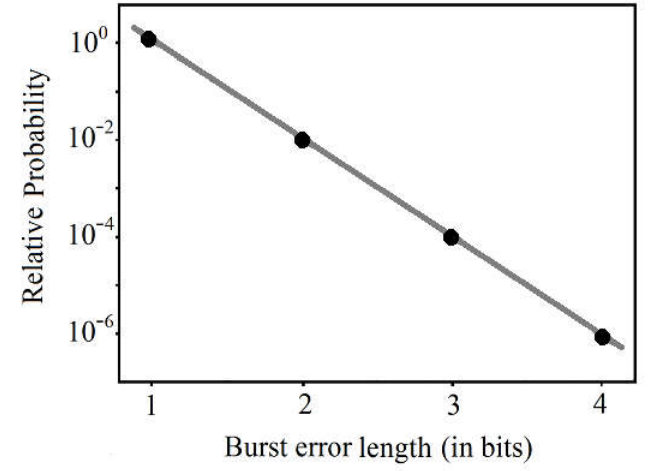

b)

Fig. 1. Optical computer networks: (a) the topology and (b) the typical error behavior.

The organization of this paper is as follows: Section 2 deals with the construction of integer double error correcting and $\mathrm{TA}_{1}$ error correcting (DEC-(TAEC) $)_{b}$ ) codes. The error control procedure for these codes is described in Section 3. Section 4 is devoted to the application of the proposed codes in OCNs, while Section 5 compares these codes with linear DEC-TAEC codes. Finally, Section 6 concludes the paper.

\section{CODES CONSTRUCTION}

Definition 1. [14] Let $Z_{2^{b-1}}=\left\{0,1, \ldots, 2^{b}-2\right\}$ be the ring of integers modulo $2^{b}-1$ and let $B_{i}=\sum_{n=0}^{b-1} a_{n} \cdot 2^{n}$ be the integer representation of a b-bit byte, where $a_{n} \in\{0,1\}$ and $1 \leq i \leq k$. Then, the code $C(b, k, c)$, defined as

$C(b, k, c)=\left\{x \in Z_{2^{b}-1}^{k+1}: \sum_{i=1}^{k} C_{i} \cdot B_{i} \equiv B_{k+1}\left(\bmod 2^{b}-1\right)\right\}$

is an $(k b+b, k b)$ integer code, where $x=\left(B_{1}, B_{2}, \ldots, B_{k}, B_{k+1}\right)$ $\in Z_{2^{b}-1}^{k+1}$ is the codeword vector, $c=\left(C_{1}, C_{2}, \ldots, C_{k}, 1\right) \in Z_{2^{b}-1}^{k+1}$ is the coefficient vector and $B_{k+1} \in Z_{2^{b}-1}$ is an integer.

Definition 2. Let $x=\left(B_{1}, B_{2}, \ldots, B_{k}, B_{k+1}\right) \in Z_{2^{b}-1}^{k+1}, y=\left(\underline{B}_{1}\right.$, $\left.\underline{B}_{2}, \ldots, \underline{B}_{k}, \underline{B}_{k+1}\right) \in Z_{2^{b}-1}^{k+1}$ and $e=\left(\underline{B}_{1}-B_{1}, \underline{B}_{2}-B_{2}, \ldots, \underline{B}_{k}-B_{k}\right.$, $\left.B_{k+1}-\underline{B}_{k+1}\right)=\left(e_{1}, e_{2}, \ldots, e_{k}, e_{k+1}\right) \in Z_{2^{b}-1}^{k+1}$ be respectively, the sent codeword, the received codeword and the error vector. Then, the syndrome $S$ of the received codeword is defined as

$S=\sum_{i=1}^{k} C_{i} \cdot \underline{B}_{i}-\underline{B}_{k+1}\left(\bmod 2^{b}-1\right)=\sum_{i=1}^{k+1} e_{i} \cdot C_{i}\left(\bmod 2^{b}-1\right)$

From (1) and (2) it can be noticed that the byte with all 1s has the same integer value as the byte with all $0 \mathrm{~s}$ (since $2^{b}-1$ $\left.\equiv 0\left(\bmod 2^{b}-1\right)\right)$. This means that we cannot construct codes that correct byte errors. However, we can construct codes with DEC-(TAEC) $)_{b}$ capability. The first step in that direction is to define sets of syndromes that correspond to single, double and $\mathrm{TA}_{1}$ errors.

Definition 3. The set of syndromes corresponding to single errors is defined as

$s_{1}=\bigcup_{i=1}^{k+1}\left\{ \pm 2^{r} \cdot C_{i}\left(\bmod 2^{b}-1\right): 0 \leq r \leq b-1\right\}$

Definition 4. The set of syndromes corresponding to double errors is defined as $s_{2}=d_{1} \cup d_{2}$

where

$$
\begin{aligned}
& d_{1}=\bigcup_{i=1}^{k+1}\left\{\left( \pm 2^{r} \pm 2^{s}\right) \cdot C_{i}\left(\bmod 2^{b}-1\right): 0 \leq r<s \leq b-1\right\} \\
& d_{2}=\bigcup_{i=1}^{k} \bigcup_{j=i+1}^{k+1}\left\{ \pm 2^{r} \cdot C_{i} \pm 2^{s} \cdot C_{j}\left(\bmod 2^{b}-1\right): 0 \leq r, s \leq b-1\right\}
\end{aligned}
$$

Definition 5. The set of syndromes corresponding to $T A_{I}$ errors is defined as

$$
s_{3}=\bigcup_{i=1}^{k+1}\left\{\left( \pm 2^{2} \pm 2^{1} \pm 2^{0}\right) \cdot 2^{r} \cdot C_{\mathrm{i}}\left(\bmod 2^{b}-1\right): 0 \leq r \leq b-3\right\}
$$

If we compare the sets $s_{1}, s_{3}$ and $d_{1}$, we will observe that their intersection is not empty. Moreover, it can be proved that the sets $s_{1}$ and $s_{3}$ are subsets of $d_{1}$.

Theorem 1. For any $b \geq 3$ it holds $s_{1} \subseteq d_{1}$ and $s_{3} \subseteq d_{1}$.

Proof. To prove that $s_{1} \subseteq d_{1}$, observe that the set $d_{1}$ can be express as the union

$d_{1}=\bigcup_{i=1}^{2 b-2} P_{i}$

where

$$
\begin{gathered}
P_{1}=\bigcup_{i=1}^{k+1}\left\{ \pm\left(2^{1}-2^{0}\right) \cdot 2^{r} \cdot C_{i}\left(\bmod 2^{b}-1\right): 0 \leq r \leq b-2\right\} \\
P_{2}=\bigcup_{i=1}^{k+1}\left\{ \pm\left(2^{1}+2^{0}\right) \cdot 2^{r} \cdot C_{i}\left(\bmod 2^{b}-1\right): 0 \leq r \leq b-2\right\} \\
P_{3}=\bigcup_{i=1}^{k+1}\left\{ \pm\left(2^{2}-2^{0}\right) \cdot 2^{r} \cdot C_{i}\left(\bmod 2^{b}-1\right): 0 \leq r \leq b-3\right\} \\
P_{4}=\bigcup_{i=1}^{k+1}\left\{ \pm\left(2^{2}+2^{0}\right) \cdot 2^{r} \cdot C_{i}\left(\bmod 2^{b}-1\right): 0 \leq r \leq b-3\right\} \\
P_{5}=\bigcup_{i=1}^{k+1}\left\{ \pm\left(2^{3}-2^{0}\right) \cdot 2^{r} \cdot C_{i}\left(\bmod 2^{b}-1\right): 0 \leq r \leq b-4\right\} \\
P_{6}=\bigcup_{i=1}^{k+1}\left\{ \pm\left(2^{3}+2^{0}\right) \cdot 2^{r} \cdot C_{i}\left(\bmod 2^{b}-1\right): 0 \leq r \leq b-4\right\} \\
\quad \vdots \\
P_{2 b-7}=\bigcup_{i=1}^{k+1}\left\{ \pm\left(2^{b-3}-2^{0}\right) \cdot 2^{r} \cdot C_{i}\left(\bmod 2^{b}-1\right): 0 \leq r \leq 2\right\} \\
P_{2 b-6}=\bigcup_{i=1}^{k+1}\left\{ \pm\left(2^{b-3}+2^{0}\right) \cdot 2^{r} \cdot C_{i}\left(\bmod 2^{b}-1\right): 0 \leq r \leq 2\right\}
\end{gathered}
$$




$$
\begin{aligned}
& P_{2 b-5}=\bigcup_{i=1}^{k+1}\left\{ \pm\left(2^{b-2}-2^{0}\right) \cdot 2^{r} \cdot C_{i}\left(\bmod 2^{b}-1\right): 0 \leq r \leq 1\right\} \\
& P_{2 b-4}=\bigcup_{i=1}^{k+1}\left\{ \pm\left(2^{b-2}+2^{0}\right) \cdot 2^{r} \cdot C_{i}\left(\bmod 2^{b}-1\right): 0 \leq r \leq 1\right\} \\
& P_{2 b-3}=\bigcup_{i=1}^{k+1}\left\{ \pm\left(2^{b-1}-2^{0}\right) \cdot 2^{r} \cdot C_{i}\left(\bmod 2^{b}-1\right): r=0\right\} \\
& P_{2 b-2}=\bigcup_{i=1}^{k+1}\left\{ \pm\left(2^{b-1}+2^{0}\right) \cdot 2^{r} \cdot C_{i}\left(\bmod 2^{b}-1\right): r=0\right\}
\end{aligned}
$$

From the above it is easy to show that

$$
\begin{aligned}
& P_{2 b-3}=\bigcup_{i=1}^{k+1}\left\{ \pm\left(2^{b-1}-1\right) \cdot C_{i}\left(\bmod 2^{b}-1\right)\right\} \equiv \\
& \equiv \bigcup_{i=1}^{k+1}\left\{\mp 2^{b-1} \cdot C_{i}\left(\bmod 2^{b}-1\right)\right\}, \\
& s_{1} \equiv P_{1} \bigcup P_{2 b-3} .
\end{aligned}
$$

Hence, it is clear that $s_{1} \subseteq d_{1}$. To prove the second part of the theorem, note that the set $s_{3}$ can be express as the union

$$
s_{3}=\bigcup_{j=1}^{8} Q_{j}
$$

where

$$
\begin{aligned}
& Q_{1}=\bigcup_{i=1}^{k+1}\left\{\left(-2^{2}-2^{1}-2^{0}\right) \cdot 2^{r} \cdot C_{i}\left(\bmod 2^{b}-1\right): 0 \leq r \leq b-3\right\} \\
& Q_{2}=\bigcup_{i=1}^{k+1}\left\{\left(-2^{2}-2^{1}+2^{0}\right) \cdot 2^{r} \cdot C_{i}\left(\bmod 2^{b}-1\right): 0 \leq r \leq b-3\right\} \\
& Q_{3}=\bigcup_{i=1}^{k+1}\left\{\left(-2^{2}+2^{1}-2^{0}\right) \cdot 2^{r} \cdot C_{i}\left(\bmod 2^{b}-1\right): 0 \leq r \leq b-3\right\} \\
& Q_{4}=\bigcup_{i=1}^{k+1}\left\{\left(-2^{2}+2^{1}+2^{0}\right) \cdot 2^{r} \cdot C_{i}\left(\bmod 2^{b}-1\right): 0 \leq r \leq b-3\right\} \\
& Q_{5}=\bigcup_{i=1}^{k+1}\left\{\left(2^{2}-2^{1}-2^{0}\right) \cdot 2^{r} \cdot C_{i}\left(\bmod 2^{b}-1\right): 0 \leq r \leq b-3\right\} \\
& Q_{6}=\bigcup_{i=1}^{k+1}\left\{\left(2^{2}-2^{1}+2^{0}\right) \cdot 2^{r} \cdot C_{i}\left(\bmod 2^{b}-1\right): 0 \leq r \leq b-3\right\} \\
& Q_{7}=\bigcup_{i=1}^{k+1}\left\{\left(2^{2}+2^{1}-2^{0}\right) \cdot 2^{r} \cdot C_{i}\left(\bmod 2^{b}-1\right): 0 \leq r \leq b-3\right\} \\
& Q_{8}=\bigcup_{i=1}^{k+1}\left\{\left(2^{2}+2^{1}+2^{0}\right) \cdot 2^{r} \cdot C_{i}\left(\bmod 2^{b}-1\right): 0 \leq r \leq b-3\right\}
\end{aligned}
$$

By comparing the sets $Q_{2}, Q_{3}, Q_{4}, Q_{5}, Q_{6}, Q_{7}, P_{1}, P_{3}$ and $P_{4}$ we conclude that

$$
\begin{aligned}
& \left(Q_{2} \cup Q_{7}\right) \equiv P_{4} \\
& \left(Q_{3} \cup Q_{6}\right) \equiv P_{3} \\
& \left(Q_{4} \cup Q_{5}\right) \subseteq P_{1} .
\end{aligned}
$$

Also, if we compare the sets $Q_{1}, Q_{8}, P_{5}$ and $P_{2 b-7}$ we see that $\left(Q_{1} \cup Q_{8}\right)=\left\{ \pm\left(2^{3}-2^{0}\right) \cdot 2^{r} \cdot C_{i}\left(\bmod 2^{b}-1\right): 0 \leq r \leq b-3\right\} \equiv$

$$
\begin{aligned}
& \equiv\left(\bigcup_{i=1}^{k+1}\left\{\left[ \pm\left(2^{3}-2^{0}\right) \cdot 2^{b-3} \cdot C_{i}\right]\left(\bmod 2^{b}-1\right)\right\} \cup P_{5}\right)= \\
& =\left(\bigcup_{i=1}^{k+1}\left\{\left[\mp\left(2^{b-3}-2^{0}\right) \cdot C_{i}\right]\left(\bmod 2^{b}-1\right)\right\} \cup P_{5}\right) \\
& \subseteq\left(P_{2 b-7} \cup P_{5}\right) .
\end{aligned}
$$

As a result, it is follows that $s_{3} \subseteq d_{1}$. $\square$
Now, when we know that $s_{1} \subseteq d_{1}$ and $s_{3} \subseteq d_{1}$, we can prove the following theorem.

Theorem 2. The codes defined by (1) can correct all single, double and $T A_{1}$ errors iff there exists $k$ mutually different coefficients $C_{i}$ such that

1. $\left|d_{1}\right|=\left[2 \cdot(b-1)^{2}-2\right] \cdot(k+1)$,

2. $\left|d_{2}\right|=2 \cdot b^{2} \cdot k \cdot(k+1)$,

3. $d_{1} \cap d_{2}=\varnothing$,

where $|A|$ is the cardinality of $A$.

Proof. Condition 1 of this theorem says that double errors confined to a $b$-bit byte generate $\left[2 \cdot(b-1)^{2}-2\right] \cdot(k+1)$ nonzero syndromes. To prove this, let us first observe that $P_{3} \subseteq P_{2}$ and $P_{2 b-5} \subseteq\left(P_{2 b-2} \cup P_{2}\right)$. Now, suppose that the coefficients $C_{i}$ have values such that

$\left|P_{2 j-1}\right|=\left|P_{2 j}\right|=2 \cdot(b-j) \cdot(k+1), j=1,2, \ldots, b-1$,

$P_{1} \cap P_{2} \cap P_{4} \cap P_{5} \cap \cdots \cap P_{2 b-6} \cap P_{2 b-4} \cap P_{2 b-3} \cap P_{2 b-2}=\varnothing$.

In that case, it is easy to show that

$\left|d_{1}\right|=\sum_{j=1}^{2 b-2}\left|P_{j}\right|-\left|P_{3}\right|-\left|P_{2 b-5}\right|=\left[2 \cdot(b-1)^{2}-2\right] \cdot(k+1)$.

Similarly, Condition 2 implies that double errors corrupting two $b$-bit bytes generate $2 \cdot b^{2} \cdot k \cdot(k+1)$ nonzero syndromes. To prove this, observe that the set $d_{2}$ can be expressed as

$$
d_{2}=\bigcup_{i=1}^{k} R_{i}
$$

where

$$
\begin{aligned}
R_{1} & =\bigcup_{j=2}^{k+1}\left\{ \pm 2^{r} \cdot C_{1} \pm 2^{s} \cdot C_{j}\left(\bmod 2^{b}-1\right): 0 \leq r, s \leq b-1\right\} \\
R_{2} & =\bigcup_{j=3}^{k+1}\left\{ \pm 2^{r} \cdot C_{2} \pm 2^{s} \cdot C_{j}\left(\bmod 2^{b}-1\right): 0 \leq r, s \leq b-1\right\} \\
R_{3} & =\bigcup_{j=4}^{k+1}\left\{ \pm 2^{r} \cdot C_{3} \pm 2^{s} \cdot C_{j}\left(\bmod 2^{b}-1\right): 0 \leq r, s \leq b-1\right\} \\
& \vdots \\
R_{k} & =\left\{ \pm 2^{r} \cdot C_{k} \pm 2^{s}\left(\bmod 2^{b}-1\right): 0 \leq r, s \leq b-1\right\}
\end{aligned}
$$

Obviously, if the coefficients $C_{i}$ have values such that $R_{1} \cap R_{2} \cap \cdots \cap R_{k}=\varnothing$,

$\left|R_{1}\right|=4 \cdot b^{2} \cdot k$,

$\left|R_{2}\right|=4 \cdot b^{2} \cdot(k-1)$,

$\left|R_{3}\right|=4 \cdot b^{2} \cdot(k-2)$,

$\left|R_{k}\right|=4 \cdot b^{2}$

then

$$
\left|d_{2}\right|=\sum_{i=1}^{k}\left|R_{i}\right|=4 \cdot b^{2} \cdot \sum_{i=1}^{k}(k+1-i)=2 \cdot b^{2} \cdot k \cdot(k+1) .
$$

Finally, Condition 3 is a necessary condition for distinguishing double errors confined to one $b$-bit byte from those corrupting two $b$-bit bytes. So, $(k b+b, k b)$ integer DEC-(TAEC) $)_{b}$ codes must satisfy all the conditions 1 to 3 . Conversely, if the codes satisfy conditions 1 to 3 , then we can distinguish double errors within one $b$-bit byte from those corrupting two $b$-bit bytes. 


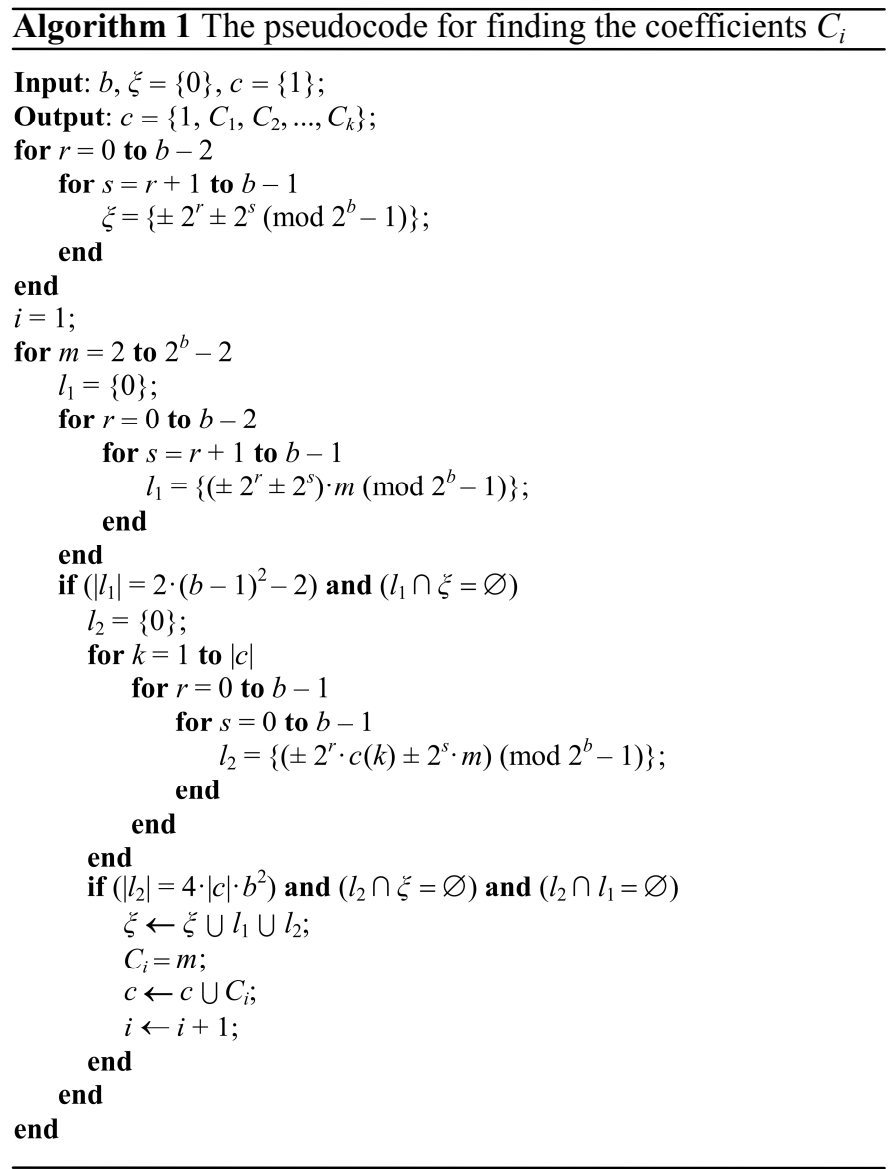

We can also correct all single, double and $\mathrm{TA}_{1}$ errors. Hence, these codes are $(k b+b, k b)$ integer DEC-(TAEC) $)_{b}$ codes. $\square$

As a corollary of Theorem 2, we can state the following.

Theorem 3. The error set for $(k b+b, k b)$ integer $D E C$ $(\mathrm{TAEC})_{b}$ codes has

$|\xi|=\left|s_{2}\right|=\left|d_{1}\right|+\left|d_{2}\right|=2 \cdot[b \cdot(k+1)-1]^{2}-2$

nonzero elements.

Proof. The proof follows from Theorem 2. $\square$

Theorem 4. For any $(k b+b, k b)$ integer DEC-(TAEC) $)_{b}$ code it holds that

$k \leq\left\lfloor\frac{2^{\frac{b-1}{2}}-b+1}{b}\right\rfloor$.

Proof. From Theorem 3 it is known that the error set $\xi$ has $2 \cdot[b \cdot(k+1)-1]^{2}-2$ nonzero elements. On the other hand, Definition 1 states that the total number of nonzero syndromes is equal to $2^{b}-2$. Obviously, if we combine Definition 1 with Theorem 3 we get the inequality

$2 \cdot[b \cdot(k+1)-1]^{2}-2 \leq 2^{b}-2$

where from it follows that

$k \leq\left\lfloor\frac{2^{\frac{b-1}{2}}-b+1}{b}\right\rfloor$

In Section 1 it was mentioned that the presented codes can be also used as high-rate ED codes. However, before showing this, we need to prove the following theorem.
TABLE I

NuMBer of COEFFICIENTS FOR SOME INTEGER DEC-(TAEC) ${ }_{b}$ CODES

\begin{tabular}{|c|c|c|c|c|c|c|c|c||}
\hline & $b=9$ & $b=10$ & $b=11$ & $b=12$ & $b=13$ & $b=14$ & $b=15$ & $b=16$ \\
\hline Theory & 0 & 1 & 2 & 2 & 4 & 5 & 7 & 10 \\
\hline Experiment & 0 & 0 & 1 & 1 & 1 & 2 & 3 & 3 \\
\hline
\end{tabular}

TABLE II

FIRST 96 COEFFICIENTS FOR SOME INTEGER DEC-(TAEC) $)_{b}$ CODES.

\begin{tabular}{|c|c|c|c|c|c|c|c|}
\hline \multicolumn{8}{|c|}{$b=16$} \\
\hline 53 & 231 & 1067 & & & & & \\
\hline \multicolumn{8}{|c|}{$b=24$} \\
\hline 45 & 201 & 477 & 1109 & 1319 & 3129 & 3453 & 4847 \\
\hline 9581 & 10117 & 11837 & 15411 & \begin{tabular}{|l|}
17897 \\
\end{tabular} & 28827 & 44061 & 52265 \\
\hline 74329 & 119841 & 174283 & 302403 & 674075 & 830035 & & \\
\hline \multicolumn{8}{|c|}{$b=32$} \\
\hline 45 & 201 & 477 & 1109 & 1319 & 3129 & 3453 & 4847 \\
\hline 9581 & 10117 & 11837 & 15411 & 17897 & 18439 & 23781 & 29749 \\
\hline 34757 & 36419 & 44865 & 46009 & 51889 & 68223 & 81619 & 93047 \\
\hline 108053 & 112279 & 113181 & 117189 & 164183 & 167119 & 169211 & 196783 \\
\hline 201311 & 209395 & 256657 & 264427 & 275489 & 282429 & 310935 & 354225 \\
\hline 386703 & 428269 & 432035 & 446911 & 514953 & 599285 & 634607 & 690403 \\
\hline \begin{tabular}{|l|}
748103 \\
\end{tabular} & 774457 & 834335 & 892067 & 893141 & 1013237 & 1067671 & 1087365 \\
\hline 1103047 & 1122449 & 1191261 & 1248189 & 1297563 & 1342681 & 1458509 & 1570785 \\
\hline 1701685 & 1789337 & 1904439 & 2007495 & 2143923 & 2240111 & 2300025 & 2332779 \\
\hline 2346995 & 2583965 & 2597467 & 3176613 & 3200875 & 3218123 & 3333741 & 3677993 \\
\hline 3759663 & 4008735 & 4245743 & 4301929 & 4539051 & 4637371 & 5001505 & 5168905 \\
\hline 5659385 & 6365959 & 7104157 & 7276563 & 8858289 & 8994505 & 9282467 & 9856101 \\
\hline
\end{tabular}

Theorem 5. Any $(k b+b, k b)$ integer DEC-(TAEC $)_{b}$ code, when operating as the ED code, is able to detect all quadruple errors, all DTA $A_{1}$ errors and all DTA $A_{2}$ errors.

Proof. From coding theory, it is known that any DEC code can detect all quadruple errors. On the other hand, from the proof of Theorem 1 we know that some double errors, within a $b$-bit byte, produce the same syndromes as $\mathrm{TA}_{1}$ errors. From this we easily conclude that $\mathrm{DTA}_{2}$ errors generate the same syndromes as some quadruple errors corrupting two $b$-bit bytes. In the same way, it can be concluded that DTA $\mathrm{D}_{1}$ errors produce the same syndromes as some quadruple errors corrupting one $b$-bit byte. Therefore, any $(k b+b, k b)$ integer DEC-(TAEC) ${ }_{b}$ code, when operating in the ED mode, is able to detect all quadruple errors, all $\mathrm{DTA}_{1}$ errors and all $\mathrm{DTA}_{2}$ errors. $\square$

To illustrate the applicability of the above theory, we have conducted an exhaustive computer search. Our first goal was to find out how the number of the $C_{i}$ 's depends on the byte length (Table I), while the second goal was to find the $C_{i}$ 's that allow us to construct some practical codes (Table II). The pseudocode of our search method is presented in Algorithm 1.

\section{ERROR CONTROL PROCEDURE}

The error control procedure for the proposed codes is very similar to those described in [9]-[14]. In short, if it operates in the ED mode, the decoder has only one task: to check whether the received data are correct or not. As a result, the incoming packet will be either dropped $(S \neq 0)$ or accepted $(S=0)$. However, if it operates in the EC mode, the decoder will try to recover the original data. The first step in this direction is to obtain the EC data from the syndrome table (ST). After that, in the next step, the decoder will execute one of the following operation(s): 
TABLE III

THE SyNdROME TABLE FOR THE $(22,11)$ INTEGER DEC-(TAEC) ${ }_{11}$ CodE.

\begin{tabular}{|c|c|c|c|c|c|c|c|c|c|c|c|c|c|c|c|c|c|}
\hline & Element of $\xi$ & $i$ & $E_{1}$ & $j$ & $E_{2}$ & & Element of $\xi$ & $i$ & $E_{1}$ & $j$ & $E_{2}$ & & Element of $\xi$ & $i$ & $E_{1}$ & $j$ & $E_{2}$ \\
\hline 1 & 1 & 2 & 1 & 0 & 0 & 291 & 640 & 2 & 1407 & 0 & 0 & 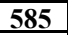 & 1395 & 1 & 2016 & 0 & 0 \\
\hline 2 & 2 & 2 & 2 & 0 & 0 & 292 & 641 & 1 & 1032 & 0 & 0 & 586 & 1396 & 1 & 512 & 2 & 1919 \\
\hline 3 & 3 & 2 & 3 & 0 & 0 & 293 & 642 & 1 & 1919 & 2 & 1023 & $\mathbf{5 8 7}$ & 1404 & 1 & 128 & 2 & 1023 \\
\hline 4 & 4 & 2 & 4 & 0 & 0 & 294 & 643 & 1 & 1919 & 2 & 1024 & 588 & 1405 & 1 & 128 & 2 & 1024 \\
\hline$\vdots$ & $\vdots$ & $\vdots$ & $\vdots$ & $\vdots$ & $\vdots$ & $\vdots$ & $\vdots$ & $\vdots$ & $\vdots$ & $\vdots$ & $\vdots$ & $\vdots$ & $\vdots$ & $\vdots$ & $\vdots$ & $\vdots$ & $\vdots$ \\
\hline 287 & 630 & 1 & 2033 & 0 & 0 & 581 & 1383 & 1 & 2039 & 2 & 1023 & 877 & 2043 & 2 & 4 & 0 & 0 \\
\hline 288 & 634 & 1 & 256 & 2 & 1919 & 582 & 1384 & 1 & 2039 & 2 & 1024 & 878 & 2044 & 2 & 3 & 0 & 0 \\
\hline 289 & 637 & 1 & 128 & 2 & 256 & 583 & 1391 & 1 & 16 & 2 & 64 & 879 & 2045 & 2 & 2 & 0 & 0 \\
\hline 290 & 639 & 1 & 32 & 2 & 32 & 584 & 1394 & 1 & 60 & 0 & 0 & 880 & 2046 & 2 & 1 & 0 & 0 \\
\hline
\end{tabular}

- for errors corrupting one b-bit byte

$$
\begin{aligned}
& B_{i}=\underline{B}_{i}+E_{1}\left(\bmod 2^{b}-1\right), 1 \leq i \leq k+1 ; \\
& E_{1} \in\left\{\mp 2^{r} \mp 2^{s}\left(\bmod 2^{b}-1\right): 0 \leq r<s \leq b-1\right\}
\end{aligned}
$$

- for errors corrupting two b-bit bytes

$$
\begin{aligned}
& B_{i}=\underline{B}_{i}+E_{1}\left(\bmod 2^{b}-1\right), 1 \leq i \leq k ; \\
& B_{j}=\underline{B}_{j}+E_{2}\left(\bmod 2^{b}-1\right), i<j \leq k+1 ; \\
& E_{1}, E_{2} \in\left\{\mp 2^{r}\left(\bmod 2^{b}-1\right): 0 \leq r \leq b-1\right\}
\end{aligned}
$$

To generate the ST it is necessary to substitute the values of $b$ and $C_{i}$ into (5)-(6). In this way, exactly $|\xi|$ (Theorem 3) relationships between the nonzero syndrome (element of $\xi$ ), error location(s) $(i, j)$ and error vector(s) $\left(E_{1}, E_{2}\right)$ will be established (Fig. 2). So, when $S \neq 0$, the decoder's task will be to find the entry with the first $b$ bits as that of the syndrome $S$. If the $\mathrm{ST}$ is sorted, this task will be finished after $n_{\mathrm{TL}}$ table lookups and $n_{\mathrm{TL}}$ comparisons $\left(1 \leq n_{\mathrm{TL}} \leq\left\lfloor\log _{2}|\xi|\right\rfloor+2\right)$ [9].

To make this procedure more clear, let us consider it on example of the $(22,11)$ DEC-(TAEC) $)_{11}$ code. (Remark: the syndrome table for this code has $|\xi|=880$ entries, and thus, it will be shown partially.)

Example 1. Let $b=11, k=1$ and $C_{1}=45$. Now, suppose that we want to transmit 9 bits of data, $B_{1}=10010011101_{2}=$ 1181. In that case, the integer value of the second (last) byte will be equal to

$B_{k+1}=B_{2}=45 \cdot 1181(\bmod 2047)=1970=11110110010_{2}$ and the codeword will have the form, $x=\left(B_{1}, B_{2}\right)=(1181$, $1970)=\left(10010011101_{2}, 11110110010_{2}\right)$.

Scenario 1: Suppose that the 6th and 11th bit are received in error, $y=\left(\underline{B}_{1}, \underline{B}_{2}\right)=\left(1001011110 \underline{0}_{2}, 11110110010_{2}\right)=(1212$, 1970). As explained previously, after calculating the value of the syndrome $S$

$S=C_{1} \cdot \underline{B}_{1}-\underline{B}_{2}(\bmod 2047)=1212 \cdot 45-1970(\bmod 2047)=1395$

the decoder will lookup the ST to find the entry with the first 11 bits as that of the syndrome $S$ (Table III). After that, in the next step, it will execute the operation

$B_{1}=1212+2016(\bmod 2047)=1181$.

Scenario 2: Assume that the 4th and 12th bit are received in error, $y=\left(\underline{B}_{1}, \underline{B}_{2}\right)=\left(100 \underline{0} 0011101_{2}, \underline{0} 1110110010_{2}\right)=(1053$, 946). As in the previous case, the decoder will calculate $S=C_{1} \cdot \underline{B}_{1}-\underline{B}_{2}(\bmod 2047)=1053 \cdot 45-946(\bmod 2047)=1405$ and conclude that the value $S=1405$ indicates an error within the first and second byte (Table III). As a result, the following procedure will take place

$B_{1}=1053+128(\bmod 2047)=1180$,

$B_{2}=946+1024(\bmod 2047)=1970$. \begin{tabular}{|l|l|r|r|r|}
\hline Element of $\xi(S)$ & Error location $(i)$ & Error vector $\left(E_{1}\right)$ & Error location $(j)$ & Error vector $\left(E_{2}\right)$ \\
\hline$-b \longrightarrow$ & - & $\left.\log _{2}(k+1)\right] \rightarrow$ & - & - \\
\hline
\end{tabular}

Fig. 2. Bit-width of one syndrome table entry.

\section{APPLICATION IN MODERN OCNS}

If we analyze the procedures described above, we will note that the decoder uses very simple operations: integer additions, integer multiplications and table lookups. As these operations are supported by all processors, it is interesting to analyze how the proposed codes can be used in modern OCNs. Without loss of generality, we will suppose that all nodes (computers, routers, etc.) are equipped with six-core processors having the same specifications as in [14] (Fig. 3a). Likewise, we will assume that each node has two modes of operation: the ED mode, in which the data are protected by the six-byte interleaved $(3104,3072)$ code, and the EC mode, in which the data are protected with the six-byte interleaved $(704,672)$ code. In that case, regardless of the mode of operation, each node will compute the values of six syndromes [14]:

- Core 1

$$
S_{1}=\sum_{i=1}^{k} C_{i} \cdot \underline{B}_{6 \cdot(i-1)+1}-\underline{B}_{6 \cdot k+1}\left(\bmod 2^{32}-1\right)
$$

- Core 2

$$
S_{2}=\sum_{i=1}^{k} C_{i} \cdot \underline{B}_{6 \cdot(i-1)+2}-\underline{B}_{6 \cdot k+2}\left(\bmod 2^{32}-1\right)
$$

\section{- Core 6}

$$
S_{6}=\sum_{i=1}^{k} C_{i} \cdot \underline{B}_{6 \cdot(i-1)+6}-\underline{B}_{6 \cdot k+6}\left(\bmod 2^{32}-1\right)
$$

The only difference is that, in the EC mode $(k=21)$, the NNs perform much less operations than in the ED mode $(k=96)$. However, when viewed from the throughput standpoint, this is not a disadvantage. Namely, from [14] we know that, in the case of operating in the EC mode, each node requires one second to decode

$G_{\mathrm{EC}}=\frac{\left(3.3 \cdot 10^{9}\right) \cdot 192 \cdot k}{9.5 \cdot k+35 \cdot n_{\mathrm{TL}}+3}$

data bits. If we apply this result to the presented theory, we can easily conclude that, for $k=21$, each node achieves a throughput of $14.19 \mathrm{Gbps}$ (Fig. 3b). On the other hand, if they operate in the ED mode, the NNs will not lookup the STs or perform any EC operation. As a result, the expression (14) reduces to 


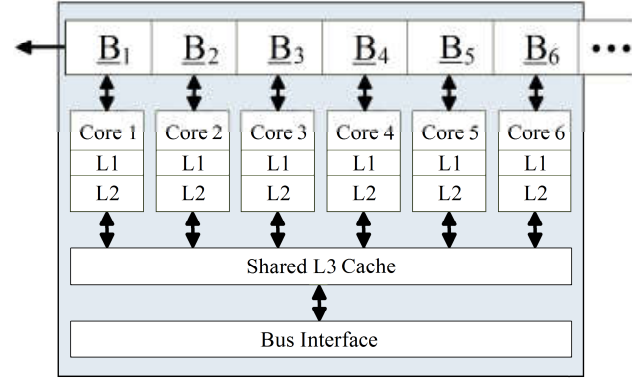

(a)

\begin{tabular}{|c|c|c|c|c|}
\hline $\begin{array}{c}\text { Codes } \\
\text { (EC mode) }\end{array}$ & $k$ & $\begin{array}{c}\text { Memory } \\
\text { Requirements } \\
\text { for Storing the } \\
\text { Syndrome Table }\end{array}$ & $n_{\text {TLmax. }}$ & $\begin{array}{c}\text { Minimum } \\
\text { Theoretical } \\
\text { Decoding } \\
\text { Throughput }\end{array}$ \\
\hline$(544,512)$ & 16 & $7.81 \mathrm{MB}$ & 21 & $11.39 \mathrm{Gbps}$ \\
\hline$(576,544)$ & 17 & $8.76 \mathrm{MB}$ & 21 & $11.97 \mathrm{Gbps}$ \\
\hline$(608,576)$ & 18 & $9.76 \mathrm{MB}$ & 21 & $12.55 \mathrm{Gbps}$ \\
\hline$(640,608)$ & 19 & $10.82 \mathrm{MB}$ & 21 & $13.11 \mathrm{Gbps}$ \\
\hline$(672,640)$ & 20 & $11.93 \mathrm{MB}$ & 21 & $13.66 \mathrm{Gbps}$ \\
\hline$(704,672)$ & 21 & $13.10 \mathrm{MB}$ & 21 & $14.19 \mathrm{Gbps}$ \\
\hline
\end{tabular}

\begin{tabular}{|c|c|c|}
\hline $\begin{array}{c}\text { Codes } \\
\text { (ED mode) }\end{array}$ & $k$ & $\begin{array}{c}\text { Theoretical } \\
\text { Decoding } \\
\text { Throughput }\end{array}$ \\
\hline$(2944,2912)$ & 91 & $66.62 \mathrm{Gbps}$ \\
\hline$(2976,2944)$ & 92 & $66.62 \mathrm{Gbps}$ \\
\hline$(3008,2976)$ & 93 & $66.62 \mathrm{Gbps}$ \\
\hline$(3040,3008)$ & 94 & $66.62 \mathrm{Gbps}$ \\
\hline$(3072,3040)$ & 95 & $66.62 \mathrm{Gbps}$ \\
\hline$(3104,3072)$ & 96 & $66.62 \mathrm{Gbps}$ \\
\hline
\end{tabular}

(b)

Fig. 3. (a) Block diagram of six-core processor and (b) theoretical decoding throughputs for some six-byte interleaved integer DEC-(TAEC) 32 codes.

$$
G_{\mathrm{ED}}=\frac{\left(3.3 \cdot 10^{9}\right) \cdot 192 \cdot k}{9.5 \cdot k+1}
$$

where from it is easy to calculate that, for $k=96$, each node achieves a throughput of $66.62 \mathrm{Gbps}$. Having this in mind, we can draw some general conclusions regarding the application of the proposed codes:

1. All considered codes have the potential to be used in $10 \mathrm{G}$ or slower OCNs (Fig. 3b). In the EC mode, they are able to protect between 3072 and 4032 bits. On the other hand, if operating in the ED mode, they can protect up to 18432 bits.

2. All considered codes are six times interleaved at the byte level. This means that the decoder can correct (detect) various types of errors, including all (double) TA errors.

3. All considered codes share the same coefficients $\left(C_{1}=\right.$ $\left.45, C_{2}=201, \ldots, C_{96}=9856101\right)$ (Table II). As a result, and depending on the application requirements, the NNs can easily switch from one mode to another. In the analyzed case, for example, the NNs only need to know whether they will use 21 (the EC mode) or 96 coefficients (the ED mode). On the basis of this information they will also know whether they need to lookup the STs or not.

\section{COMPARISON WITH LINEAR DEC-TAEC CODES}

In the coding literature, one can find only two codes with DEC-TAEC capability [15], [16]. The first was obtained by modifying the DEC-BCH codes and the second by modifying the DEC-OLS codes. In both cases, the modifications were done at the cost of increased hardware complexity, but without adding additional check bits.

As for the DEC-TAEC BCH codes, they have parameters $\left(2^{u}-1,2^{u}-1-2 \cdot u\right)$, where $u \geq 4$. This means that the codes of highest-rate are $(15,7),(31,21)$ and so on. On the other hand, it is known that the DEC-OLS codes are very inefficient in terms of redundancy. The parameters of these codes are $\left(\mathrm{m}^{2}\right.$ $\left.+4 \cdot m, m^{2}\right)$, where $m \geq 1$, which means that they require many more check bits than the DEC-TAEC BCH codes. Unlike the linear DEC-TAEC codes, the proposed ones are characterized by the parameters $(k b+b, k b)$. Although there is a significant difference between experimental and theoretical results (Table I), it can be said that the proposed codes are relatively efficient in terms of redundancy. This is also seen from the fact that for data lengths up to 2048 bits they require 3 to 6 check bits more compared to the DEC-TAEC BCH codes, i.e. 9 to 154 checkbits less than the DEC-TAEC OLS codes (Table IV).

When it comes to the processing of data bits, the difference between the mentioned codes is even more pronounced. First
TABLE IV

CHeCK-Bit Lengths of THE Proposed AND Linear DEC-TAEC Codes.

\begin{tabular}{||c|c|c|c|c|c|c|c||}
\hline \hline \multirow{2}{*}{ Codes } & \multicolumn{7}{|c||}{ Data word length (bits) } \\
\cline { 2 - 9 } & 32 & 64 & 128 & 256 & 512 & 1024 & 2048 \\
\hline Codes from [15] & 12 & 14 & 16 & 18 & 20 & 22 & 24 \\
\hline Proposed codes & 15 & 17 & 20 & 22 & 25 & 27 & 30 \\
\hline Codes from [16] & 24 & 32 & 48 & 64 & 92 & 128 & 184 \\
\hline \hline
\end{tabular}

TABLE V

COMPARISON OF THE PROPOSED CODES AND LINEAR DEC-TAEC CODES.

\begin{tabular}{||c|c|c|c||}
\hline $\begin{array}{c}\text { Main } \\
\text { characteristics }\end{array}$ & $\begin{array}{c}\text { DEC-TAEC } \\
\text { BCH codes }\end{array}$ & $\begin{array}{c}\text { Proposed } \\
\text { codes }\end{array}$ & $\begin{array}{c}\text { DEC-TAEC } \\
\text { OLS codes }\end{array}$ \\
\hline $\begin{array}{c}\text { Error correction } \\
\text { capabilities }\end{array}$ & $\begin{array}{c}\text { Correction of } \\
\text { single, double } \\
\text { and TA errors }\end{array}$ & $\begin{array}{c}\text { Correction of } \\
\text { single, double } \\
\text { and TA } \text { A }_{b 1} \text { errors }\end{array}$ & $\begin{array}{c}\text { Correction of } \\
\text { single, double } \\
\text { and TA errors }\end{array}$ \\
\hline $\begin{array}{c}\text { Processing of } \\
\text { data bits }\end{array}$ & $\begin{array}{c}\text { Finite field } \\
\text { operations }\end{array}$ & $\begin{array}{c}\text { Integer and } \\
\text { LUT operations }\end{array}$ & $\begin{array}{c}\text { Finite field } \\
\text { operations }\end{array}$ \\
\hline Interleaving & $\begin{array}{c}\text { Causes } \\
\text { extra delay } \\
\text { and requires } \\
\text { additional } \\
\text { hardware }\end{array}$ & $\begin{array}{c}\text { Do not cause } \\
\text { extra delay and } \\
\text { do not require } \\
\text { additional } \\
\text { hardware }\end{array}$ & $\begin{array}{c}\text { Causes } \\
\text { extra delay } \\
\text { and requires } \\
\text { additional } \\
\text { hardware }\end{array}$ \\
\hline $\begin{array}{c}\text { Adaptation } \\
\text { to the application } \\
\text { requirements }\end{array}$ & Very complex & Simple & Complex \\
\hline $\begin{array}{c}\text { Preferred type of } \\
\text { implementation }\end{array}$ & Hardware & Software & Hardware \\
\hline
\end{tabular}

of all, we have seen that the proposed codes use integer and LUT operations, which makes them suited for implementation on modern processors (Table V). Moreover, the analysis from Section 4 showed that they have the potential to achieve throughputs of several tens of Gbps. This result would be even better if multiple integer units (instead of one) per core were used (since Haswell all Intel processors have four integer units per core [17]). On the other hand, it is known that the linear DEC-TAEC codes use FF operations to encode/decode data bits. However, as these operations are not supported by GPPs, the codes from [15], [16] must be implemented in dedicated hardware (the software-based decoders need several tens of clock cycles to process one bit [18], [19]). In this regard, it should be noted that the DEC-TAEC OLS codes have several advantages over the DEC-TAEC BCH codes. One is that they can be decoded much faster than the DEC-TAEC BCH codes. Another advantage is that the DEC-TAEC OLS decoder can be implemented in modular form. This means that each added module provides extra correction ability allowing the receiver to switch between different EC modes. Although this feature is similar to that of the proposed codes, it cannot be efficiently exploited in modern OCNs. The reasons for this lie in the high 
complexity of the decoder (it is necessary to process up to 12000 data bits) as well as high redundancy of the DECTAEC OLS codes.

Finally, the last difference between the mentioned codes concerns the error control capabilities. As shown in Section 2, the proposed codes were originally designed to correct all single, double and $\mathrm{TA}_{1}$ errors. However, in Section 4 we saw that they have the ability to be interleaved without delay and without using any additional hardware. Thanks to this, it is possible to construct codes capable of detecting/correcting (multiple) random and TA errors. In addition, these codes can easily change the code rate and the mode of operation, which is very useful when transmitting different types of data. On the other hand, the linear DEC-TAEC codes have the ability to correct single, double and TA errors, but cannot easily change the code rate and/or the mode of operation. More precisely, they could be used for that purpose if different codecs were integrated into one chip. This would, however, lead to a significant increase in the price of network equipment. The similar applies to the use of interleaved codes, which are further characterized by delays in the processing of data bits.

\section{CONCLUSION}

In this paper, we presented a class of integer codes that are suitable for use in optical computer networks where the data is transmitted serially. The presented codes use integer and table lookup operations, which makes them well suited for software implementation. Besides this, the presented codes have the ability to change the code rate and the mode of operation. In the error correction mode, which is suitable for real-time applications, the decoder can correct all single and double errors as well as all triple-adjacent errors within one $b$-bit byte. On the other hand, in the error detection mode, which is suited for non real-time applications, the decoder can detect all quadruple errors, all double triple-adjacent errors within one $b$-bit byte and all double triple-adjacent errors within two $b$-bit bytes. Finally, the proposed codes can be interleaved without delay and without using any additional hardware. Owing to this, it is possible to construct very simple codes capable of correcting (detecting) various types of errors, including all (double) triple-adjacent errors.

\section{REFERENCES}

[1] R. Ramaswani, K. Sivarajan and G. Sasaki, Optical Networks: A Practical Perspective, 3rd ed., Elsevier, Inc., 2010.

[2] P. Ciccarelli et al., Networking Basics, 2nd ed., John Wiley \& Sons, Inc., 2013.

[3] R. Giladi, Network Processors: Architecture, Programming, and Implementation, Elsevier, Inc., 2008.

[4] Observations of Error Characteristics of Fiber Optic Transmission Systems, CCITT Study Group XVIII Contrib. D21, San Diego, CA, USA, Jan. 1989.

[5] W. D. Grover, "Effect of Error Correcting Code Using DS3 Framing Bits on Measured Dribble Error Pattern of $565 \mathrm{Mb} / \mathrm{s}$ Fibre Optic Transmission System," Elect. Lett., vol. 28, no. 20, pp. 1869-1870, Sept. 1992.

[6] T. Ono et al., "Bit Error Statistical Analysis of Optical Transmission Systems," in D.W. Faulkner and A.L. Harmer (Eds.), pp. 43-49, IOS Press, 2000.

[7] D. Mello, E. Offer, and J. Reichert, "Error Arrival Statistics for FEC Design in Four-Wave Mixing Limited Systems," in Proc. Opt. Fiber Commun. Conf. (OFC), Mar. 2003, pp. 529-530.
[8] L. James, "Error Behaviour in Optical Networks," Ph.D. dissertation, Dept. Eng., Univ. Cambridge, Cambridge, U.K., 2005.

[9] A. Radonjic and V. Vujicic, "Integer Codes Correcting Burst Errors within a Byte," IEEE Trans. Comput., vol. 62, no. 2, pp. 411-415, Feb. 2013.

[10] A. Radonjic and V. Vujicic, "Integer Codes Correcting Spotty Byte Asymmetric Errors," IEEE Commun. Lett., vol. 20, no. 12, pp. 23382341, Dec. 2016.

[11] A. Radonjic and V. Vujicic, "Integer Codes Correcting High-Density Byte Asymmetric Errors," IEEE Commun. Lett., vol. 21, no. 4, pp. 694697, Apr. 2017.

[12] A. Radonjic, "(Perfect) Integer Codes Correcting Single Errors," IEEE Commun. Lett., vol. 22, no. 1, pp. 17-20, Jan. 2018.

[13] A. Radonjic and V. Vujicic, "Integer Codes Correcting Burst and Random Asymmetric Errors within a Byte," J. Franklin Inst., vol. 355, no. 2, pp. 981-996, Jan. 2018.

[14] A. Radonjic and V. Vujicic, "Integer Codes Correcting Sparse Byte Errors," Cryptogr. Commun., vol. 11, no. 5, pp. 1069-1077, Sept. 2019.

[15] W. Wu et al., "Method of Correcting Adjacent Errors By Using BCHBased Error Correction Coding," US 2013/0262957 A1, Oct. 3, 2013.

[16] S. Liu et al., "Reducing the Cost of Triple Adjacent Error Correction in Double Error Correction Orthogonal Latin Square Codes," IEEE Trans. Device Mater. Rel., vol. 16, no. 2, pp. 269-271, Jun. 2016.

[17] A. Fog, "The Microarchitecture of Intel, AMD and via CPUs: An Optimization Guide for Assembly Programmers and Compiler Makers," Technical University of Denmark, Mar. 8, 2020. [Online]. Available: https://www.agner.org/optimize/microarchitecture.pdf

[18] J. Cho and W. Sung, "Efficient Software-Based Encoding and Decoding of BCH Codes," IEEE Trans. Comput., vol. 58, no. 7, pp. 878-889, July 2009.

[19] A. Subbiah and T. Ogunfunmi, "A Flexible Hybrid BCH Decoder for Modern NAND Flash Memories Using GPGPUs," Micromachines, vol. 10, no. 6, pp. 1-15, Jun. 2019. 